\title{
Minimally Invasive Valve Surgery via Right Mini-Thoracotomy: Technical Aspects to Facilitate Prosthetic Valve Insertion*
}

\author{
Mohammed Hassan ${ }^{1 \#}$, Tomas A. Salerno ${ }^{2}$, Jimmy Windsor ${ }^{3}$, Marco Ricci ${ }^{1}$ \\ ${ }^{1}$ Division of Cardiothoracic Surgery, University of New Mexico Health Science Center, Albuquerque, USA \\ ${ }^{2}$ Division of Cardiothoracic Surgery, University of Miami Miller School of Medicine/Jackson Memorial Hospital, Miami, USA \\ ${ }^{3}$ Department of Anesthesiology, University of New Mexico Health Science Center, Albuquerque, USA \\ Email: ${ }^{\#}$ MHassan@salud.unm.edu
}

Received July 17, 2013; revised August 15, 2013; accepted August 29, 2013

Copyright (C) 2013 Mohammed Hassan et al. This is an open access article distributed under the Creative Commons Attribution License, which permits unrestricted use, distribution, and reproduction in any medium, provided the original work is properly cited.

\begin{abstract}
Mitral and tricuspid valve surgery is increasingly being performed through a right mini-thoracotomy approach. Although valve repair is preferred over replacement whenever possible, valve replacement may be required in certain patients. In situations where the mitral or tricuspid anatomy are unfavorable and exposure is difficult, seating a prosthetic valve in the annulus through a right mini-thoracotomy approach may be difficult, compared to conventional sternotomy approach. This is complicated by limited tactile feedback in the minimally invasive approach. Herein, we describe several simple maneuvers that facilitate proper prosthetic valve seating and visualization of the posterior annulus during minimally invasive valve operations via right mini-thoracotomy.
\end{abstract}

Keywords: Mitral Valve Surgery; Minimally Invasive Mitral Valve Surgery; Techniques in Mitral Valve Surgery

\section{Introduction}

Mitral and tricuspid valve operations are increasingly performed through a right mini-thoracotomy approach [1-3]. Although valve repair remains the preferred option over replacement, in some situations mitral or tricuspid prosthetic valve replacement may be required [3]. In conventional valve operations through a sternotomy approach, prosthetic valve insertion and seating in the native valve annulus are easily accomplished as the surgeon has broad direct visual and tactile feedback. Conversely, in minimally invasive valve replacement through a right mini-thoracotomy approach, exposure and tactile feedback are limited. In situations where exposure of the mitral or tricuspid annulus is unfavorable, maintaining the prosthetic valve seated during knot tying and verifying proper seating particularly at the level of the posterior valve annulus may be difficult. Improper positioning of the valve prosthesis may result in longer ischemic time due to the need of placing additional repair sutures or repair paravalvular leaks. Herein, we describe several simple maneuvers that facilitate proper prosthetic valve

\footnotetext{
*The authors have no disclosures in relation to this research.

\#Corresponding author.
}

seating and visualization of the posterior annulus during minimally invasive valve operations via right mini-thoracotomy.

\section{Surgical Technique}

After a right mini-thoracotomy through the fourth intercostal space is performed, exposure of the mitral or tricuspid valve is obtained by opening the respective atrial chamber. The operative details of the operations as they relate to cardiopulmonary bypass strategy and exposure of the valve have been well described previously [4]. If valve replacement is needed in the setting of mitral or tricuspid valve surgery, we prefer to use annular sutures with the pledgets placed on the atrial side, as this facilitates confirmation of proper apposition of the annulus to the sewing ring of the valve after the valve is implanted.

Following insertion of the sutures through the annulus of the valve (mitral or tricuspid), the sutures are passed though the sewing ring of the prosthesis, and the prosthesis is glided down into the native annulus. The valve handle is released. At this stage, especially if a tissue valve is used, proper orientation of the struts and proper insertion of the prongs of the prosthesis through the annulus is 


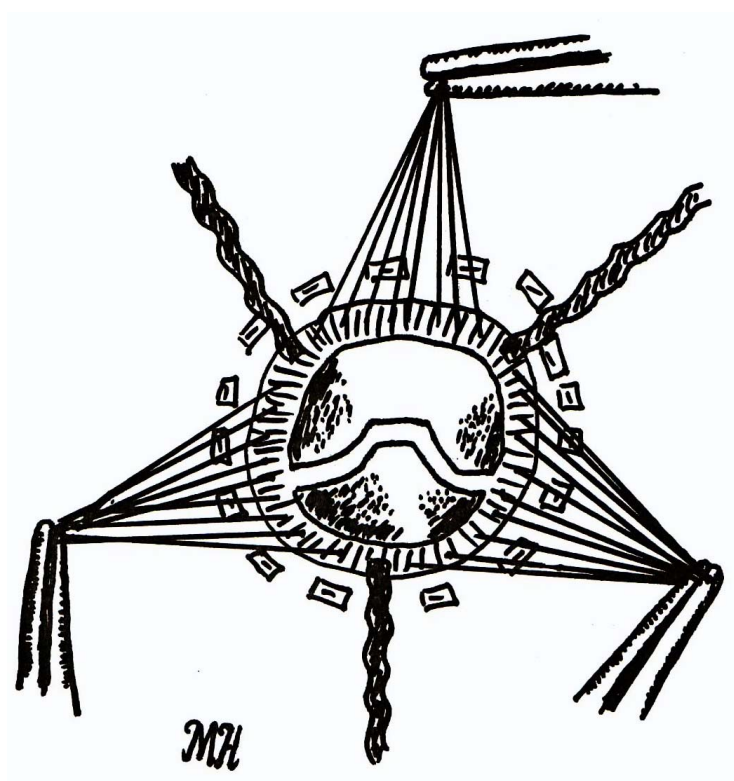

Figure 1. Valve seated in the annulus and secured by 3 snared red rubber Rummel's tourniquets (red rubber).

critical. We recommend true-sizing the prosthesis to avoid insertion difficulty into the annulus. During knot tying with a knot pusher, the prosthesis may dislodge from its seated position as the surgeon does not have the ability to push the valve down as effectively as conventional surgery via sternotomy, where the valve is pushed down in the annulus with each throw. During minimally invasive surgery, this problem may be avoided by placing three individual snares (or tourniquet) to secure the three commissural sutures of the prosthesis, thereby maintaining the prosthesis in a well seated position when tying the rest of the sutures (Figure 1). After all sutures are tied, the three snares are released and the three remaining valve sutures are tied. At this stage, proper position of the prosthesis within the annulus is confirmed by examining the cuff position relative to the suture pledgets. However, confirmation of proper valve positioning may still be difficult at the level of the posterior annulus in certain cases of unfavorable valve annular geometry and orientation. In such cases, it is useful to use a long dental mirror to visualize the posterior annulus and confirm proper valve placement.

\section{Discussion}

Mitral or tricuspid valve replacement is increasingly performed through a minimally invasive right mini-thoracotomy approach. Using this approach, the surgeon has limited tactile feedback. In situations of unfavorable atrial anatomy and annular geometry, proper mitral or tricuspid prosthetic valve positioning and seating may be challenging. Improper prosthetic valve seating requires cumbersome surgical maneuvers to repair paravalvular leaks and may considerably delay the operative time. We present a technique in the minimally invasive approach of using three tourniquets to stabilize the prosthesis at the annular level to enhance proper prosthetic valve positioning, and to minimize the risk of a paravalvular leak.

\section{Conclusion}

The presented technique with tourniquets is effective to assist in seating prosthetic valves in the Mitral or Tricuspid minimally invasive surgeries.

\section{REFERENCES}

[1] J. Seeburger, M. A. Borger, J. Passage, et al., "Minimally Invasive Isolated Tricuspid Valve Surgery,” The Journal of Heart Valve Disease, Vol. 19, No. 2, 2010, pp. 189192.

[2] T. C. Lee, B. Desai and D. D. Glower, "Results of 141 Consecutive Minimally Invasive Tricuspid Valve Operations: An 11-Year Experience," The Annals of Thoracic Surgery, Vol. 88, No. 6, 2009, pp. 1845-1850. doi:10.1016/j.athoracsur.2009.08.052

[3] J. Seeburger, M. A. Borger, V. Falk, et al., "Minimal Invasive Mitral Valve Repair for Mitral Regurgitation: Results of 1339 Consecutive Patients,” European Journal Cardio-Thoracic Surgery, Vol. 34, No. 4, 2008, pp. 760765. doi:10.1016/j.ejcts.2008.05.015

[4] J. Seeburger, M. A. Borger, N. Doll, et al., "Comparison of Outcomes of Minimally Invasive Mitral Valve Surgery for Posterior, Anterior and Bileaflet Prolapse,” European Journal Cardio-Thoracic Surgery, Vol. 36, No. 3, 2009, pp. 532-538. doi:10.1016/j.ejcts.2009.03.058 